\section{Are $\mathrm{N}$-acetylcysteine and adalimumab effective for non-alcoholic steatohepatitis?}

\author{
Mustafa Yalçın, ${ }^{1}$ Muhammed Ali Kıyak, ${ }^{1}$ \\ Mesut Akarsu, ${ }^{1}$ Aslı Çelik, ${ }^{2}$ Göksel Bengi, ${ }^{1}$ \\ Özgül Sağol ${ }^{3}$ \\ 1Department of Gastroenterology, \\ 2Department of Animal Laboratory, \\ ${ }^{3}$ Department of Pathology, Dokuz Eylül \\ University, İzmir, Turkey
}

\section{Abstract}

Due to the lack of effective medical treatment for non-alcoholic steatohepatitis (NASH), we aimed to evaluate new treatment options. In particular, our goal was to investigate and compare the effects of $\mathrm{N}$-acetylcysteine (NAC) and Adalimumab treatment on tumor necrosis factor alpha (TNF- $\alpha$ ) and oxidative stress during the development of NASH in a rat model of the disease. Our study included a total of 35 female Wistar albino rats that were divided into 5 groups of 7 each, and evaluated over a 6 week period. One group received a normal diet, while the other four groups received a methionine and choline deficient (MCD) diet. One of the groups receiving the MCD diet did not take any medicine, while the other three were administered NAC, adalimumab, or a NAC/adalimumab combination therapy. NASH was successfully established in the MCD diet group. Levels of TNF- $\alpha$ were effectively suppressed in the three groups that received therapy. Even though adalimumab significantly enhanced suppression of TNF- $\alpha$, the NASH score was suppressed to a more statistically significant extent in the groups receiving NAC. Our study showed that TNF- $\alpha$ and oxidative stress play an important role in NASH pathogenesis. The antioxidant agent, NAC, was found to be superior to the anti-TNF agent, Adalimumab, in the improvement of total NASH score. Although these drugs did not prevent the development of $\mathrm{NASH}$, it was shown that they mildly reverse the NASH histopathology score, suggesting improvement of and overall liver function.

\section{Introduction}

Non-alcoholic fatty liver diseases (NAFLD) cover a wide spectrum of liver damage ranging from NASH, to fibrosis and cirrhosis, and to steatosis. ${ }^{1} \mathrm{NASH}$ development during the natural course of disease is caused by inflammation, fibrosis, and cirrhosis. The pathogenesis of NASH is very complex and is typically initiated by insulin resistance. Further progressive damage to the liver is necessary to cause steatohepatitis the most severe form of NAFLD. It has been suggested that the interaction between oxidative stress and cytokines plays a key role in NASH. The increased production of Adalimumab treatment on tumor necrosis factor alpha (TNF- $\alpha$ ) has been observed in non-alcoholic steatohepatitis (NASH) rat models. Increased serum TNF- $\alpha$ concentration has also been reported in humans with $\mathrm{NASH}^{2-4}$

A methionine and choline deficient (MCD) diet impairs the synthesis of phosphatidylinositol choline, which is required for synthesis of very-low-density lipoprotein (VLDL). In phosphatidylinositol choline deficiency, serum triglyceride levels are decreased while liver triglyceride levels are increased. As a result, steatosis occurs. ${ }^{5,6}$ An MCD diet displaces hepatic oxidants such as glutathione (GSH) and S-adenozilmetionin (S-AME) ${ }^{7}$ and increases oxidative stress by suppressing oxidative defense mechanisms. ${ }^{8}$ This cascade of events induces TNF- $\alpha$ and other proinflammatory cytokines $^{9}$ that contribute to NAFLD and insulin resistance with similar mechanisms. Therefore, MCD model studies are very informative because they emphasize the importance of oxidant stress in the pathogenesis of hepatic steatosis and steatohepatitis (independent of obesity). Aminotransferase levels, histological changes in the liver characterized by steatosis, focal inflammation, necrosis of hepatocytes, and fibrosis are induced in mice fed with the MCD diet. These rapid histological changes in morphology are similar to those seen in human patients with NASH. ${ }^{10,11}$

Lipid-lowering agents such as statins and fibrates, ${ }^{12,13}$ antioxidants such as vitamin E, ${ }^{14-16}$ and cytoprotective agents such as ursodeoxycholic acid (UDCA) have been shown to reduce the level of aminotransferase. In addition, they were shown to improve liver histology in several studies. ${ }^{17}$ However, none of these drugs specifically target NASH. Therefore, there is a need for specific drugs that target and treat NASH.

Adalimumab is a human monoclonal antibody that specifically recognizes TNF- $\alpha$. It has been used to treat diseases such as rheumatoid arthritis and Crohn's disease. In a previous case report, Schramm and colleagues demonstrated that the use of adalimumab for rheumatoid arthritis returned aspartate aminotransferase (AST), alanine aminotransferase (ALT), and gamma glutamyl transferase (GGT) levels back to normal in patients with $\mathrm{NASH}^{9}$

$\mathrm{N}$-acetylcysteine (NAC) is a product of cysteine metabolism and was initialized as a mucolytic agent in the treatment of chronic lung disease in 1963. It is a precursor for the
Correspondence: Göksel Bengi, Department of Gastroenterology, Dokuz Eylül University Faculty of Medicine, İzmir, Turkey

Tel.: +905324626972.

E-mail: drgokselbengi@hotmail.com

Key words: Non-alcoholic steatohepatitis; $\mathrm{N}$-acetylcysteine; Adalimumab; tumor necrosis factor alpha.

Contributions: the authors contributed equally.

Conflict of interest: the authors declare no potential conflict of interest.

Received for publication: 1 January 2016. Accepted for publication: 18 January 2017.

This work is licensed under a Creative Commons Attribution NonCommercial 4.0 License (CC BYNC 4.0).

(C) Copyright M. Yalçın et al., 2016

Licensee PAGEPress, Italy

Gastroenterology Insights 2016; 7:6227

doi:10.4081/gi.2016.6227

major endogenous antioxidant glutathione and has been used as an antidote in acetaminophen toxicity more than forty years. Glutathione is a tri-peptide that includes cysteine and it functions in cellular events such as amino acid transport and scavenging of free radicals, intermediate reactive oxygen products, and alkaline toxic chemicals. ${ }^{18}$ Nakano and colleagues observed that steatotic rats had significantly lower levels of reduced glutathione in their liver when compared to nonsteatotic rats. The same study showed increasing levels of reduced glutathione in steatotic rats after NAC administration. ${ }^{19}$ NAC is also known for its function related to microcirculatory relaxation. ${ }^{20}$ In steatotic liver, the sinusoidal space was reported to decrease by $54.0 \%$ when compared to the normal liver, and hepatic blood flow was shown to be limited to $1 / 3$ of normal. ${ }^{21}$ Currently, there are no effective options for medical treatment for NASH. Since TNF- $\alpha$ and oxidative stress play an important role in NASH, we aimed to investigate and compare the effects of NAC and TNF- $\alpha$ and oxidative stress during the development of $\mathrm{NASH}$ in a rat model of the disease.

\section{Materials and Methods}

This study was approved by the Dokuz Eylul University Ethics Committee on April 11, 2012

\section{Animals}

A total of 35 female Wistar albino rats weighing between 194-250 g (average $223.6 \mathrm{~g}$ ) 
were obtained from the Experimental Research Laboratory, Faculty of Medicine, Dokuz Eylul University Hospital. Rats were put into cages and maintained according to the international animal experimentation and maintenance manual under standard laboratory conditions (temperature $23 \pm 2{ }^{\circ} \mathrm{C}, 12$-hour day/night cycle).

\section{Diet}

Rats were given either a normal diet or an MCD diet. The content and chemical structure was standardized by animal diet experts (Harlan Teklad, Madison, WI, USA). The rats were given $10 \mathrm{~g} / 100 \mathrm{~g}$ food and 10-12 mL/100 $\mathrm{g}$ water daily.

\section{Study design}

Thirty-five female Wistar albino rats were divided into five equal groups of 7 rats in each group.

Group 1 ( $n=7$, control): The control group. Rats were fed with standard food and water.

Group 2 ( $n=7, M C D)$ : Received an MCD diet for 6 weeks.

Group $3(n=7, M C D+N A C)$ : Administered 20 $\mathrm{mg} / \mathrm{kg}$ NAC per day by orogastric gavage and were fed with an MCD diet and water for 6 weeks. These doses were based on previously published findings. ${ }^{22}$

Group 4 ( $n=7, \quad$ MCD+adalimumab): Received $7 \mathrm{mg} / \mathrm{kg}$ adalimumab intraperitoneally at weeks 0,2 , and 4 and were fed and MCD diet and water for 6 weeks. The dose of $7 \mathrm{mg} / \mathrm{kg}$ adalimumab was described previously. ${ }^{23}$

Group 5 ( $n=7, M C D+N A C+$ adalimumab): The rats in this group were administered 20 $\mathrm{mg} / \mathrm{kg}$ NAC per day by orogastric gavage and received $7 \mathrm{mg} / \mathrm{kg}$ adalimumab intraperitoneally at weeks 0,2 , and 4 . Group five were fed with an MCD diet and water for 6 weeks.

\section{Termination of the experiment and collecting samples}

Six weeks after initiation of the study, the abdomen was opened under ether anesthesia and approximately $10 \mathrm{cc}$ blood was drawn from the inferior vena cava. Blood samples were centrifuged at $5000 \mathrm{rpm}$ for 5 minutes and stored at $-80^{\circ} \mathrm{C}$ until analysis. Liver tissues were placed in $10.0 \%$ formalin and were sent to the pathology laboratory for histopathological examinations. Rats were sacrificed by ether inhalation.

\section{Cytokine levels}

TNF- $\alpha$ (Invitrogen, KRC3011, USA), TGF , IL-6 (Boster, EK0514, China) and IL-8 (Cusabio Int, CSB-E07273r, China) levels were measured by ELISA.

\section{Biochemical analysis}

Aspartate aminotransferase (AST), alanine aminotransferase (ALT), alkaline phosphatase (ALP), total bilirubin, total protein, and albumin levels were assessed by using Abbott Architect 16000 autoanalyzer (Abbott Inc., Princeton, NJ, USA).

\section{Histopathologic evaluation}

Liver tissue samples were obtained and fixed in paraffin blocks for processing using traditional methods for histological examination. The liver was cut for hematoxylin-eosin staining. The prevalence of fibrosis was determined by using Mason Trichrome. An expert pathologist examined the samples under a light microscope. The presence or absence of steatosis, ballooning degeneration, lobular inflammation, and fibrosis were assessed and Brunt NASH scoring was performed. Scores of five and above were considered diagnostic for NASH.

\section{Statistics}

SPSS 15.0 computer software (SPSS Inc., Sostware Chicago, IL, USA) was used for statistical analysis. Differences between the groups were determined by Kruskal-Wallis and MannWhitney U tests and P-values of less than 0.05 were considered statistically significant.

\section{Results}

Rats from each group were weighed weekly starting from the first day of study. The group that was fed a normal rat diet had significant weight gain. The four groups receiving MCD diet showed weight loss, with the highest weight loss occurring in the group that received MCD diet only. Average weights of the groups according to weeks are depicted in Figure 1. No side effect was observed in any of the rats throughout the study period.

\section{Immunological parameters}

Mean serum levels of TNF- $\alpha$, TGF , IL6, and IL8 in all groups are presented in Table 1. Parameters showing statistically significant differences between groups were determined with Kruskal-Wallis test. Those that were found to be statistically significant were com-

Table 1. Cytokine levels.

\begin{tabular}{|c|c|c|c|c|c|c|c|}
\hline $\begin{array}{l}\text { Immunological } \\
\text { parameters }\end{array}$ & $\begin{array}{c}\text { All rats } \\
(\mathrm{n}=35) ; \\
\text { mean (SD) }\end{array}$ & $\begin{array}{l}\text { Normal diet } \\
\qquad(\mathrm{n}=7) ; \\
\text { mean (SD) }\end{array}$ & $\begin{array}{c}\text { MCD } \\
(n=7) ; \\
\text { mean (SD) }\end{array}$ & $\begin{array}{c}\mathrm{MCD}+\mathrm{NAC} \\
(\mathrm{n}=7) ; \\
\text { mean (SD) }\end{array}$ & $\begin{array}{l}\mathrm{MCD}+\mathrm{ADA} \\
(\mathrm{n}=7) ; \\
\text { mean }(\mathrm{SD})\end{array}$ & $\begin{array}{c}\mathrm{MCD}+\mathrm{NAC}+\mathrm{ADA} \\
(\mathrm{n}=7) ; \\
\text { mean }(\mathrm{SD})\end{array}$ & P* \\
\hline TNF $\alpha(\mathrm{pg} / \mathrm{mL})$ & $3.80(3.71)$ & $1.70(2.59)$ & $8.60(3.53)$ & $4.87(3.97)$ & $1.82(0.60)$ & $2.02(1.15)$ & 0.002 \\
\hline TGF $\beta(p g / m L)$ & $29.60(15.14)$ & $15.87(3.80)$ & $50.93(13.92)$ & $27.87(8.70)$ & $29.63(11.82)$ & $23.68(8.90)$ & $<0.001$ \\
\hline IL6 (pg/mL) & $301.88(123.90)$ & $157.10(19.75)$ & $412.60(84.85)$ & $382.02(118.50)$ & $314.56(110.50)$ & $243.09(51.65)$ & $<0.001$ \\
\hline IL8 (pg/mL) & $147.14(107.39)$ & $86.96(69.24)$ & $179.10(145.88)$ & $187.01(127.92)$ & $142.04(68.44)$ & $140.60(104.29)$ & 0.381 \\
\hline
\end{tabular}

*Kruskal-Wallis Test. MCD, Methionine choline deficient; ADA, Adalimumab; NAC, N-acetylcysteine.

Table 2. Statistical data for cytokine levels among groups.

\begin{tabular}{|c|c|c|c|c|c|c|}
\hline \multirow[t]{2}{*}{ Groups } & \multicolumn{2}{|c|}{ TNF alpha } & \multicolumn{2}{|c|}{ TGF beta } & \multicolumn{2}{|c|}{ IL6 } \\
\hline & $\mathrm{Z}$ & P* & $\mathrm{Z}$ & P* & $\mathrm{Z}$ & P* \\
\hline Normal Diet vs MCD & -2.875 & 0.004 & -3.130 & 0.002 & -3.130 & 0.002 \\
\hline MCD vs MCD+NAC & -1.663 & 0.096 & -2.686 & 0.007 & -0.831 & 0.406 \\
\hline $\mathrm{MCD}$ us $\mathrm{M} M C D+\mathrm{ADA}$ & -2.747 & 0.006 & -2.435 & 0.015 & -1.663 & 0.96 \\
\hline MCD vs M MCD+NAC+ADA & -2.747 & 0.006 & -2.814 & 0.005 & -3.130 & 0.002 \\
\hline $\mathrm{MCD}+\mathrm{NAC}$ vs M MCD+ADA & -1.981 & 0.048 & -0.128 & 0.898 & -1.214 & 0.225 \\
\hline $\mathrm{MCD}+\mathrm{NAC}$ vs $\mathrm{M} \mathrm{MCD}+\mathrm{NAC}+\mathrm{ADA}$ & -1.853 & 0.064 & -1.214 & 0.225 & -2.364 & 0.018 \\
\hline $\mathrm{MCD}+\mathrm{ADA}$ us $\mathrm{M} \mathrm{MCD}+\mathrm{NAC}+\mathrm{ADA}$ & -0.064 & 0.949 & -1.151 & 0.250 & -1.469 & 0.142 \\
\hline
\end{tabular}

*Mann-Whitney U Test. MCD, Methionine choline deficient; ADA, Adalimumab; NAC, N-acetylcysteine. 
pared between the groups using MannWhitney U test (Table 2).

\section{TNF- $\alpha$ levels}

TNF- $\alpha$ levels were determined as 0.99 (0.137.42) $\mathrm{pg} / \mathrm{mL}$ in the group receiving normal diet, $9.90(2.23-11.64) \mathrm{pg} / \mathrm{mL}$ in the group receiving MCD diet only, 3.75 (1.97-13.14) $\mathrm{pg} / \mathrm{mL}$ in the group receiving $\mathrm{MCD}+\mathrm{NAC}, 1.90$ (1.09-2.58) $\mathrm{pg} / \mathrm{mL}$ in the group receiving MCD+adalimumab, and $1.33(1.12-4.24) \mathrm{pg} / \mathrm{mL}$ in the group receiving $\mathrm{MCD}+\mathrm{NAC}+$ adalimum$a b$, respectively. TNF- $\alpha$ levels were highest in group 2 , and lowest in group 1 .

\section{Biochemical parameters}

Mean serum levels of AST, ALT, ALP, total bilirubin, total protein and albumin from all groups are presented in Table 3. Parameters showing statistically significant difference between groups were determined with Kruskal-Wallis test. Those that were found to be statistically significant were compared between the groups by using Mann-Whitney U test (Table 4).

\section{Histopathology}

Hepatic steatosis, lobular inflammation, hepatocyte ballooning, fibrosis and total NASH scores for all groups are presented in Table 5 . Parameters that show statistically significant difference between the groups were determined with Kruskal Wallis test. Since the group receiving normal diet had a $0 \mathrm{NASH}$ score, this parameter was not taken into evaluation with Kruskal Wallis test. Hepatocyte ballooning and total NASH score showed statistically significant difference, and were compared between groups using Mann Whitney U test (Table 6). As expected, development of NASH was histopathologically confirmed in the group receiving MCD diet. Total score of 5 or above was accepted as NASH. When total NASH scores were evaluated among the groups, the highest total score was in the group receiving $\mathrm{MCD}+\mathrm{ADA}$ with $6.71 \pm 1.06$, while the lowest score was observed in the group receiving $\mathrm{MCD}+\mathrm{ADA}+\mathrm{NAC}$ with $5.00 \pm 1.15$. There were statistically significant differences between the groups with respect to total NASH score $(\mathrm{P}=0.032)$. When $\mathrm{MCD}+\mathrm{NAC}$ and MCD+ADA groups were compared for improvement in total NASH score, there was statistically significant difference in favor of $\mathrm{MCD}+\mathrm{NAC}$ group $(\mathrm{P}=0.037)$. When $\mathrm{MCD}+\mathrm{NAC}+\mathrm{ADA}$ and $\mathrm{MCD}+\mathrm{ADA}$ groups were compared, there was statistically significant difference in favor of MCD+NAC+ADA group $(\mathrm{P}=0.007)$. However, when the group receiving MCD diet only was compared to other groups receiving treatment, there was no statistically significant difference with respect to improvement in NASH histopathological score. As for hepatocyte ballooning, the highest score was in the group receiving MCD with $1.71 \pm 0.76$; and the lowest score was in the group receiving $\mathrm{MCD}+\mathrm{ADA}+\mathrm{NAC}$ with $0.71 \pm 0.49$. There

Table 3. Biochemical parameters.

\begin{tabular}{|c|c|c|c|c|c|c|c|}
\hline $\begin{array}{l}\text { Biochemical } \\
\text { parameters }\end{array}$ & $\begin{array}{l}\text { All rats } \\
(\mathrm{n}=35) ; \\
\text { mean }(\mathrm{SD})\end{array}$ & $\begin{array}{l}\text { Normal diet } \\
\qquad(\mathrm{n}=7) ; \\
\text { mean (SD) }\end{array}$ & $\begin{array}{c}\text { MCD } \\
(n=7) ; \\
\text { mean }(\mathrm{SD})\end{array}$ & $\begin{array}{l}\mathrm{MCD}+\mathrm{NAC} \\
(\mathrm{n}=7) \\
\text { mean }(\mathrm{SD})\end{array}$ & $\begin{array}{l}\mathrm{MCD}+\mathrm{ADA} \\
(\mathrm{n}=7) ; \\
\text { mean }(\mathrm{SD})\end{array}$ & $\begin{array}{c}\mathrm{MCD}+\mathrm{NAC}+\mathrm{ADA} \\
(\mathrm{n}=7) \\
\text { mean }(\mathrm{SD})\end{array}$ & P* \\
\hline AST (U/L) & $123(40.93)$ & $134.14(66.16)$ & $139.86(42.54)$ & 115.72 (32.67) & $112.71(14.28)$ & 112.57 (35.27) & 0.594 \\
\hline ALT (U/L) & $56.77(20.29)$ & $44.71(19.68)$ & $62.71(29.75)$ & $58(14.25)$ & $63.57(12.23)$ & $54.86(20.90)$ & 0.314 \\
\hline ALP (U/L) & $392.89(206.60)$ & $472.43(337.30)$ & $429.29(137.69)$ & 468.57 (212.43) & $314.29(122.10)$ & $279.86(106.64)$ & 0.283 \\
\hline Total Bilirubin (mg/dL) & $0.40(0.10)$ & $0.28(0.02)$ & $0.41(0.10)$ & $0.42(0.12)$ & $0.43(0.09)$ & $0.46(0.10)$ & 0.005 \\
\hline Total Protein (gr/dL) & $6.71(0.61)$ & $7.6(0.57)$ & $6.67(0.38)$ & $6.36(0.36)$ & $6.5(0.37)$ & $6.44(0.47)$ & 0.002 \\
\hline Albumin (gr/dL) & $2.99(0.49)$ & $3.69(0.43)$ & $2.79(0.46)$ & $2.80(0.30)$ & $2.79(0.27)$ & $2.89(0.25)$ & 0.006 \\
\hline
\end{tabular}

*Kruskal-Wallis Test. MCD, Methionine choline deficient; NAC, N-acetylcysteine; ADA, Adalimumab.

Table 4. Statistical data for the biochemical parameters among the groups.

\begin{tabular}{|c|c|c|c|c|c|c|}
\hline \multirow[t]{2}{*}{ Groups } & \multicolumn{2}{|c|}{ Total Bilirubin } & \multicolumn{2}{|c|}{ T. protein } & \multicolumn{2}{|c|}{ Albumin } \\
\hline & $\mathrm{Z}$ & P* & $\mathrm{Z}$ & P* & $\mathrm{Z}$ & P* \\
\hline Normal Diet vs MCD & -2.564 & 0.010 & -2.830 & 0.005 & -2.628 & 0.009 \\
\hline $\mathrm{MCD}$ vs $\mathrm{MCD}+\mathrm{NAC}$ & 0.000 & 1.000 & -1.489 & 0.136 & -0.780 & 0.435 \\
\hline MCD vs MCD+ADA & -0.319 & 0.749 & -0.712 & 0.476 & -0.581 & 0.561 \\
\hline $\mathrm{MCD}$ us $\mathrm{MCD}+\mathrm{NAC}+\mathrm{ADA}$ & -0.448 & 0.654 & -1.160 & 0.246 & -0.966 & 0.334 \\
\hline $\mathrm{MCD}+\mathrm{NAC}$ us MCD+ADA & -0.193 & 0.847 & -0.837 & 0.403 & -0.517 & 0.605 \\
\hline $\mathrm{MCD}+\mathrm{NAC}$ v $\mathrm{MCD}+\mathrm{NAC}+\mathrm{ADA}$ & -0.704 & 0.482 & -0.193 & 0.847 & 0.771 & 0.441 \\
\hline $\mathrm{MCD}+\mathrm{ADA}$ os $\mathrm{MCD}+\mathrm{NAC}+\mathrm{ADA}$ & -0.512 & 0.609 & -0.321 & 0.748 & -0.853 & 0.393 \\
\hline
\end{tabular}

*Mann-Whitney U Test. MCD, Methionine choline deficient; NAC, N-acetylcysteine; ADA, Adalimumab.

Table 5. Histopathological scores.

\begin{tabular}{|c|c|c|c|c|c|c|c|}
\hline $\begin{array}{l}\text { Histopathological } \\
\text { scores }\end{array}$ & $\begin{array}{c}\text { All rats } \\
(\mathrm{n}=35) ; \\
\text { mean (SD) }\end{array}$ & $\begin{array}{l}\text { Normal diet } \\
\qquad(n=7) ; \\
\text { mean (SD) }\end{array}$ & $\begin{array}{c}\text { MCD } \\
(n=7) ; \\
\text { mean (SD) }\end{array}$ & $\begin{array}{l}\mathrm{MCD}+\mathrm{NAC} \\
\quad(n=7) ; \\
\operatorname{mean}(\mathrm{SD})\end{array}$ & $\begin{array}{l}\mathrm{MCD}+\mathrm{ADA} \\
\quad(\mathrm{n}=7) \\
\text { mean }(\mathrm{SD})\end{array}$ & $\begin{array}{c}\mathrm{MCD}+\mathrm{NAC}+\mathrm{ADA} \\
(\mathrm{n}=7) \\
\text { mean }(\mathrm{SD})\end{array}$ & $\mathbf{P} * *$ \\
\hline Steatosis & $2.06(1.19)$ & $0(0)$ & $2.57(0.79)$ & $2.43(0.79)$ & $2.86(0.38)$ & $2.29(0.95)$ & 0.567 \\
\hline Lobular inflammation & $1.69(1.02)$ & $0(0)$ & $2(0.81)$ & $1.57(0.79)$ & $2.57(0.53)$ & $2(0.58)$ & 0.096 \\
\hline Hepatocyte ballooning & $1.14(0.73)$ & $0(0)$ & $1.71(0.76)$ & $1.57(0.53)$ & $1.29(0.49)$ & $0.71(0.49)$ & 0.029 \\
\hline Fibrosis & $0(0)$ & $0(0)$ & $0(0)$ & $0(0)$ & $0(0)$ & $0(0)$ & 1.000 \\
\hline Total NASH score & $4.86(2.30)$ & $0(0)$ & $6.14(1.07)$ & $5.57(0.98)$ & $6.71(0.76)$ & $5(1.15)$ & 0.032 \\
\hline
\end{tabular}

**Kruskal-Wallis Test (Normal diet group is not included in the analysis). MCD, Methionine choline deficient; NAC, N-acetylcysteine; ADA, Adalimumab. 
was a statistically significant difference between the groups $(\mathrm{P}=0.015)$. As for steatosis and lobular inflammation, there was no statistically significant difference between the groups. Histopathological findings are shown in Figure 2.

\section{Discussion and Conclusions}

In this study, the NASH model was successfully achieved with administration of MCD diet. At the end of six weeks, TNF- $\alpha$ levels were significantly higher statistically in the second group, indicating that levels of TNF- $\alpha$ increased in the NASH-induced group.

When serum TGF $\beta$ and IL 6 levels were evaluated, there was a statistically significant difference between the groups receiving normal diet and MCD only, which had a higher association with the second group. This showed us that TGF $\beta$ and IL 6 levels increased in NASH-induced group. As for IL 8 levels, it increased more than 2 fold in the group receiving MCD diet when compared to the group receiving normal diet; however, there was no statistically significant difference between the two groups. When biochemical parameters were evaluated, there were elevations in AST and ALT levels in the group receiving MCD compared to the group receiving normal diet. However, there was no statistically significant difference. ALP level was higher in the group receiving normal diet compared to the group receiving MCD diet, which was not statistically significant. Total bilirubin, total protein, and albumin levels were significantly higher statistically in the group receiving MCD diet.

During histopathological examination, the total NASH score was found to be zero in the group that received normal diet. NASH development was histopathologically confirmed in the MCD group. Fibrosis was not observed in the NASH-induced group. Similar to previous studies, we showed that MCD diet increased proinflammatory cytokines that have been blamed responsible for the pathogenesis of NASH development, and it successfully induced NASH development. In a previous study with rat NASH models, NASH was established by giving $100.0 \%$ high-fat diet for 6 weeks. Thong-Ngam and colleagues showed that $20 \mathrm{mg} / \mathrm{kg} /$ day peroral administration of NAC significantly increased levels of antiinflammatory acting serum glutathione, and caused regression in steatosis and necrosisinflammation score in rats fed with high-fat diet. ${ }^{22}$ Baumgardner and colleagues found that administration of $2 \mathrm{~g} / \mathrm{kg} /$ day of NAC decreased levels of ALT, TNF- $\alpha$ mRNA, and cytochrome P450 2E1 in steatosis as well as the total NASH score and porta//lobular fibrosis in NASH that was induced by total enteral polyunsaturated fat nutrition. ${ }^{24}$ In a study by Gülbahar and colleagues, there were no improvements in serum AST, ALT and GGT levels in 11 patients with NASH upon a 5.5 months follow-up with diet. After the diet, patients were given 1000 $\mathrm{mg} /$ day NAC treatment perorally. Statistically, serum AST, ALT, and GGT levels were determined to regress significantly. ${ }^{25}$ In our study, TNF- $\alpha$ was found to be suppressed in the group receiving NAC when serum TNF- $\alpha$ levels were compared between the group receiving MCD diet and the group receiving MCD+NAC.

Table 6. Statistical data for the biochemical parameters among the groups.

\begin{tabular}{lcccc} 
Groups & \multicolumn{2}{c}{ Hepatocyte ballooning } & \multicolumn{2}{c}{ Total NASH score } \\
& $\mathbf{Z}$ & $\mathbf{P} *$ & $\mathbf{Z}$ & $\mathbf{P} *$ \\
MCD vs MCD+NAC & -0.286 & 0.775 & -0.876 & 0.381 \\
MCD us MCD+ADA & -1.164 & 0.244 & -1.214 & 0.225 \\
\hline MCD vs MCD+NAC+ADA & -2.422 & 0.015 & -1.615 & 0.106 \\
MCD+NAC us MCD+ADA & -1.041 & 0.298 & -2.089 & 0.037 \\
\hline MCD+NAC us MCD+NAC+ADA & -2.442 & 0.015 & -0.876 & 0.381 \\
MCD+ADA us MCD+NAC+ADA & -1.927 & 0.054 & -2.676 & 0.007 \\
\hline
\end{tabular}

*Mann-Whitney U Test. MCD, Methionine choline deficient; NAC, N-acetylcysteine; ADA, Adalimumab.

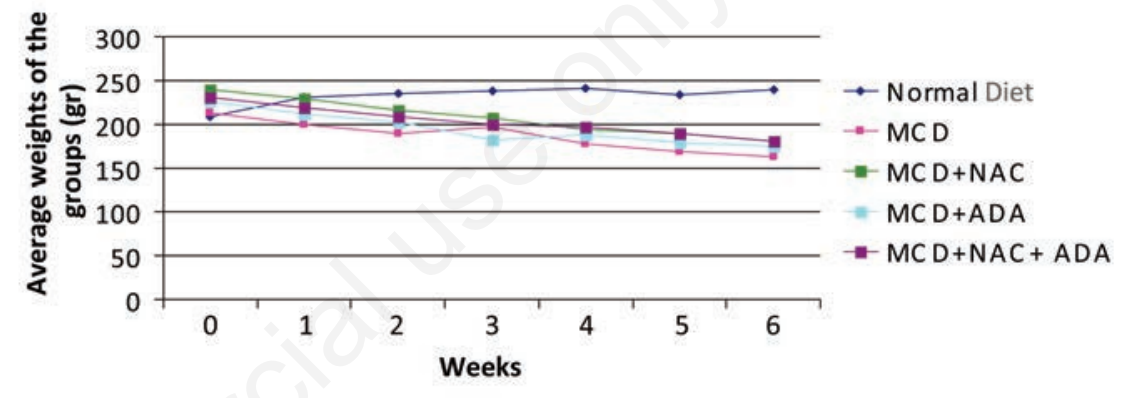

Figure 1. Average weights in the groups. MCD, Methionine and choline deficient; NAC, $\mathrm{N}$-acetylcysteine; ADA, Adalimumab.

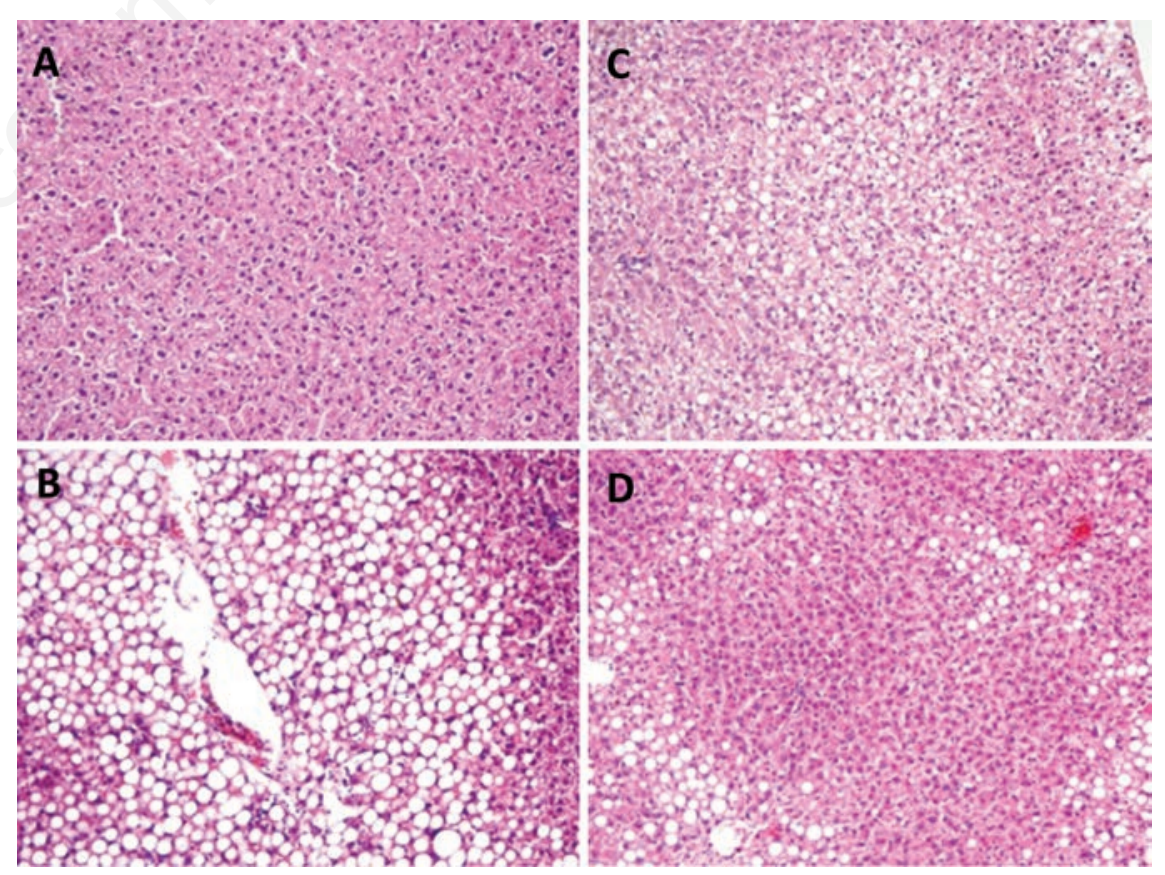

Figure 2. Normal liver tissue specimen: no steatosis, lobular inflammation or ballooning (A). Marked steatosis and lobular inflammation in the group receiving Methionine and choline deficient (MCD) diet (B). Marked hepatocyte ballooning in the group receiving MCD diet (C). Mild steatohepatitis findings in the group receiving dual treatment (D). Hematoxylin and Eosin, 200x. 
However, the difference was statistically not significant. IL6 and TGF $\beta$ levels were found to be reduced in the NAC branch compared to MCD branch. The reduction in TGF $\beta$ levels was determined to be statistically significant. IL8 levels were higher compared to other groups, but the difference was not statistically significant. Histopathological examination in the group receiving NAC revealed improvement in steatosis, lobular inflammation, hepatocyte ballooning and total NASH score; however, this improvement was statistically not significant when compared with the MCD receiving group.

There were no statistically significant differences between the groups receiving MCD and $\mathrm{MCD}+\mathrm{NAC}$ with respect to biochemical parameters (AST, ALT, ALP, bilirubin, albumin and total protein). There was a mild reduction in AST and ALT levels in the group receiving NAC. In their case report, Schramm and colleagues reported that AST, ALT, and GGT levels regressed back to normal in a patient with NASH after administration of adalimumab for rheumatoid arthritis. ${ }^{9}$ Histopathological examination was not carried out in their case. In our previously published study, adalimumab suppressed TNF- $\alpha$ and led to improvements in liver function tests and NASH score; although, this improvement was limited. We observed slightly better results with adalimumab compared to pentoxifylline and infliximab. We concluded that suppression of TNF- $\alpha$ only was not sufficient in treatment of $\mathrm{NASH}$, and that pathogenesis of NASH was multifactorial. ${ }^{23}$

In the present study, serum TNF- $\alpha$ levels were compared between the group receiving MCD diet and the group receiving MCD+adalimumab. Statistically, TNF- $\alpha$ levels were significantly suppressed in the adalimumab treated group. When the group receiving adalimumab was compared to the group receiving MCD, there was only improvement in hepatocyte ballooning score, which was not statistically significant. There was no statistically significant difference in the biochemical parameters (AST, ALT, ALP, bilirubin, albumin, and total protein) between the groups receiving MCD and MCD+ADA. However, there was slight reduction in AST and ALP levels. When compared to the group receiving MCD, there were improvements in steatosis, hepatocyte ballooning, and total NASH scores in the group receiving adalimumab+NAC; while only the improvement in hepatocyte ballooning was determined to be statistically significant. When groups receiving $\mathrm{MCD}+\mathrm{NAC}$ and $\mathrm{MCD}+\mathrm{ADA}$ were compared, there was greater suppression in steatosis, lobular inflammation, and total NASH score in the group receiving MCD+NAC. The suppression in total NASH score was determined to be statistically significant.

In conclusion, this present study showed that suppression of TNF- $\alpha$ could not prevent development of NASH, and it also did not have a remarkable effect in the treatment. However, anti-oxidant acting NAC suppressed the total NASH score significantly and greater than adalimumab. This finding suggests that the pathogenesis of NASH is multifactorial and that TNF- $\alpha$ blockage has limited benefits in the treatment of NASH. Nevertheless, further studies are needed to investigate NASH pathogenesis and the benefits of different synchronous treatment options on the disease development.

\section{References}

1. Ludwig J, Viggiano TR, McGill DB, Oh BJ. Nonalcoholic steatohepatitis: Mayo Clinic experiences with a hitherto unnamed disease. Mayo Clin Proc 1980;55:434-8.

2. Copaci I, Micu, L, Voiculescu M. The role of cytokines in non-alcoholic steatohepatitis: a systematic review. J Gastrointestin Liver Dis 2006;15:363-73.

3. Koteish A, Diehl AM. Animal models of steatohepatitis. Best Pract Res Clin Gastroenterol 2002;16:679-90.

4. Mehta K, Van Thiel DH, Shah N, Mobarhan S. Nonalcoholic fatty liver disease: pathogenesis and the role of antioxidants. Nutr Rev 2002;60:289-93.

5. Atzeni F, Sarzi-Puttini P, Doria A, et al. Potential off-label use of infliximab in autoimmune and nonautoimmune diseases: a review. Autoimmun Rev 2005;4:144-52.

6. Markham A, Lamb HM. Infliximab: a review of its use in the management of rheumatoid arthritis. Drugs 2000;59:134159 .

7. Iimuro Y, Gallucci RM, Luster MI, et al. Antibodies to tumor necrosis factor alfa attenuate hepatic necrosis and inflammation caused by chronic exposure to ethanol in the rat. Hepatology 1997;26:1530-7.

8. Tilg H, Jalan R, Kaser A, Davies NA. Antitumor necrosis factor-alpha monoclonal antibody therapy in severe alcoholic hepatitis. J Hepatol 2003;38:419-25.

9. Schramm C, Schneider A, Marx A, Lohse AW. Adalimumab could suppress the activity of non alcoholic steatohepatitis (NASH). Z Gastroenterol 2008;46:1369-71.

10. Ghoshal AK, Ahluwalia M, Farber E. The rapid induction of liver cell death in rats fed a choline-deficient methionine-low diet. Am J Pathol 1983;113:309-14.

11. Zabel P, Schade FU, Schlaak M. Inhibition of endogenous TNF formation by pentoxifylline. Immunobiology 1993;187:447-63.

12. Diehl AM. Cytokine regulation of liver injury and repair. Immunol Rev 2000;174:160-71.

13. Hensley K, Koteke Y, Sang H, Pye QN.
Dietary choline restriction causes complex I dysfunction and increased $\mathrm{H}(2) 0(2)$ generation in liver mitochondria. Carcinogenesis 2000;21:983-9.

14. Rinella ME, Elias MS, Smolak RR, et al. Mechanisms of hepatic steatosis in mice fed a lipogenic methionine choline-deficient diet. J Lipid Res 2008;49:1068-76.

15. McClain CJ, Prakash S, Mokshagundam L, et al. Mechanisms of non-alcoholic steatohepatitis. Alcohol 2004;34:67-79.

16. Rinella ME, Green RM. The methioninecholine deficient dietary model of steatohepatitis does not exhibit insulin resistance. J Hepatol 2004;40:47-51.

17. Barbuio R, Milanski M, Bertolo MB, et al. Infliximab reverses steatosis and improves insulin signal transduction in liver of rats fed a high-fat diet. J Endocrinol 2007;194:539-50.

18. Buckley NA, Whyte IM, O'Connell DL, Dawson AH. Oral or intravenous N-acetylcysteine: which is the treatment of choice for acetaminophen (paracetamol) poisoning? J Toxicol Clin Toxicol 1999;37:759-67.

19. Nakano H, Nagasaki H, Barama A, Boudjema K. The effects of N-acetylecysteine and anti-intercellular adhesion molecule-1 monoclonal antibody against ischemia-reperfusion injury of the rat steatotic liver produced by a cholinemethionine deficient diet. Hepatology 1997;26:670-8.

20. Stamler J, Mandelsohn ME, Amarante P, Smick D. N-acetyl cysteine potantiates platelet inhibition by endothelium-derived relaxing factor. Circ Res 1989;65:789-95.

21. Ghoshal AK, Farber E. Choline deficiency, lipotrope deficiency and the development of liver disease including liver cancer: a new perspective. Lab Invest 1993;68:25560.

22. Thong-Ngam D, Samuhasaneeto S, Kulaputana 0, Klaikeaw N. N-acetylcysteine attenuates oxidative stress and liver pathology in rats with non-alcoholic steatohepatitis. World J Gastroenterol 2007;13:5127-32.

23. Yalçın M, Akarsu M, Çelik A, et al. A comparison of the effects of infliximab, adalimumab, and pentoxifylline on rats with non-alcoholic steatohepatitis. Turk J Gastroenterol 2014;25:167-75.

24. Baumgardner JN, Shankar K, Hernings L, et al. N-acetylcysteine attenuates progression of liver pathology in a rat model of nonalcoholic steatohepatitis. J Nutr 2008; 138:1872-9.

25. Gulbahar 0, Karasu Z, Ersoz G. Treatment of nonalcoholic steatohepatitis with $\mathrm{N}$ acetylcysteine [abstract]. Gastroenterology 2000;118:A1444. 